\title{
Disordered XY model: Effective medium theory and beyond
}

\author{
I. Maccari, ${ }^{1}$ L. Benfatto, ${ }^{1}$ and C. Castellani ${ }^{2}$ \\ ${ }^{1}$ ISC-CNR and Department of Physics, Sapienza University of Rome, P.le A. Moro 2, 00185 Rome, Italy \\ ${ }^{2}$ Department of Physics, Sapienza University of Rome, P.le A. Moro 2, 00185 Rome, Italy
}

(Received 27 November 2018; published 11 March 2019)

\begin{abstract}
We study the effect of uncorrelated random disorder on the temperature dependence of the superfluid stiffness in the two-dimensional classical XY model. By means of a perturbative expansion in the disorder potential, equivalent to the $T$-matrix approximation, we provide an extension of the effective-medium-theory result able to describe the low-temperature stiffness and its separate diamagnetic and paramagnetic contributions. These analytical results provide an excellent description of the Monte Carlo simulations for two prototype examples of uncorrelated disorder. Our findings offer an interesting perspective on the effects of quenched disorder on longitudinal phase fluctuations in two-dimensional superfluid systems.
\end{abstract}

DOI: 10.1103/PhysRevB.99.104509

\section{INTRODUCTION}

Despite its original formulation in terms of planar spins, the XY model has been extensively investigated in the literature in the context of superconducting (SC) systems, which belong to the same universality class. From one side, the quantum XY model properly describes the Josephson-like interactions between SC grains in artificial arrays [1]. From the other side, even for homogeneous superconductors phase fluctuations at low temperature can be effectively described by coarse-grained XY-like models [2-6]. Within this context the equivalent of the Heisenberg interaction $J$ for spins becomes the energy scale connected to phase fluctuations, i.e., the superfluid stiffness $J_{s}=n_{s}^{2 D} / 4 m$, where $n_{s}^{2 D}=n_{s}^{3 d} d$ is the effective two-dimensional superfluid density, and the length scale $d$ is the smallest between the SC coherence length and the sample thickness. In the specific case of quasi-towdimensional (quasi-2D) systems, as thin SC films, the 2D XY model allows also for a proper description of the topological phase transition due to the unbinding of vortexlike excitations, as described by the Berezinskii-Kosterlitz-Thouless (BKT) theory [7-9].

While in conventional clean superconductors $J$ is a much larger energy scale than the SC gap $\Delta$, making phasefluctuation effects usually irrelevant, the suppression of $J$ in disordered superconductors or in unconventional ones brings back the issue of their possible role. In particular, it has been proven experimentally [10-15] that thin films of conventional superconductors at the verge of the superconductor-insulator transition (SIT) display a finite gap $\Delta$ above $T_{c}$, suggesting that the phase transition is driven by phase coherence more than pairing [16-21]. Moreover, at strong disorder the SC ground state itself shows an emergent inhomogeneity $[11,14,22-25]$, leading to a granular SC landscape. These findings suggest a mapping of the SC problem into an effective disordered bosonic system [26,27], whose phase degrees of freedom are conveniently described by quantum disordered XY models. The underlying inhomogeneity triggers interesting effects, such as the contribution of longitudinal quantum phase modes to the anomalous subgap optical absorption [28-32] as observed near the SIT [33-39] or in films of nanoparticles [32,40,41]. The nontrivial space structure of disorder can also have remarkable effects on the behavior of transverse (vortexlike) fluctuations in the classical limit, as we have recently investigated by means of Monte Carlo simulations $[42,43]$. Indeed, by modeling the emergent granularity of the SC landscape with space-correlated inhomogeneous local couplings, we have shown that the anomalous nucleation of vortices in the bad SC regions can lead to a substantial smearing of the BKT superfluid-stiffness jump at the transition, in agreement with the systematically broadened jumps observed experimentally in thin films of both conventional [44-48] and unconventional [49-51] superconductors. Apart from the smearing of the BKT jump, in Ref. [42] it was observed that disorder can affect the low-temperature behavior of the superfluid stiffness in a nontrivial way, depending both on the variance of the disorder probability distribution and on its spatial correlations.

These findings call for a deeper investigation of the general role of low-temperature phase fluctuations in classical disordered XY models. Here we address this problem by combining Monte Carlo simulations with an analytical diagrammatic expansion. We start with the XY Hamiltonian:

$$
H_{X Y}=-\sum_{i, \mu=\hat{x}, \hat{y}} J_{i}^{\mu} \cos \left(\theta_{i}-\theta_{i+\mu}\right),
$$

where disorder is encoded in the local couplings $J_{i}^{\mu}$. We consider two possible types of spatially uncorrelated disorder. The first one is the case of a Gaussian distribution, which is usually employed to mimic relatively weak fluctuations of the local stiffnesses around a given mean value. The second one is the diluted model, where a fraction $p$ of the couplings is taken to be equal to zero, mimicking the local suppression of the Josephson coupling between neighboring SC regions due to disorder. Despite being a model without specific spatial correlations for the disorder, its SC properties are, nonetheless, ultimately dominated by the global phenomenon 
of percolation [52]. By means of Monte Carlo simulations we compute the temperature dependence of the superfluid stiffness for increasing disorder. These results are compared with the analytical derivation of the low-temperature stiffness obtained from the calculation of the self-energy corrections to the phase propagator due to disorder. By resumming in the disorder, within the on-site $T$-matrix scheme [53], we derive a self-consistence equation for the stiffness that is formally equivalent to the results obtained in the effective-mediumtheory approximation (EMA) [28,52]. This approach allows us to generalize the EMA result to include finite-temperature corrections. The derived analytical formulas are in excellent agreement with the Monte Carlo simulations and allow one to capture the role played by different disorder models for the thermal activation of longitudinal phase fluctuations.

The plan of the paper is the following. In Sec. II, we briefly review the standard results expected for the clean XY model. In Sec. III, we consider the disordered case. After reviewing the equivalence with the random-resistor-network problem, we first solve the zero-temperature case (Sec. III A), recovering the analogy between the $T$-matrix resummation in the disorder potential and the EMA not only for the global stiffness but also for the separate diamagnetic and paramagnetic contributions. In Sec. III B, we use the same strategy to derive a modified EMA equation able to include the finitetemperature corrections to the stiffness in the presence of disorder. In Sec. IV, we compare these analytical expressions with the numerical results obtained by means of Monte Carlo simulations. Section V contains the closing discussion and remarks.

\section{TEMPERATURE DEPENDENCE OF THE STIFFNESS IN THE CLEAN CASE}

Before discussing the role of disorder, we start by briefly recalling the standard results expected for the clean $X Y$ model. We will thus consider the model (1) on an $L \times L=N$ two-dimensional square lattice with homogeneous couplings $J_{i}^{\mu}=J$. The superfluid stiffness is the response to a transverse gauge field, which can be minimally coupled to the SC phase by the replacement

$$
H_{X Y}=-J \sum_{i, \mu=\hat{x}, \hat{y}} \cos \left(\theta_{i}-\theta_{i+\mu}+A_{\mu}\right)
$$

As usual, due to the periodic boundary conditions, applying a constant field $\mathbf{A}$ along, say, the $x$ direction is equivalent to considering twisted boundary conditions for the phase, with a total flux $\phi=A_{x} L$ (in units of $\Phi_{0}=h c / 2 e$ for the SC case) through the sample. The current density is defined as $I_{x}=-N^{-1} \partial H / \partial A_{x}$, so that at leading order in $A_{x}$ one has

$$
\begin{aligned}
I_{x} & =-\frac{J}{N} \sum_{i} \sin \left(\theta_{i}-\theta_{i+\hat{x}}+A_{x}\right) \\
& \simeq-\frac{J}{N} \sum_{i}\left[\sin \left(\theta_{i}-\theta_{i+\hat{x}}\right)+\cos \left(\theta_{i}-\theta_{i+\hat{x}}\right) A_{x}\right],
\end{aligned}
$$

where the first term defines the paramagnetic current and the second one is the diamagnetic response. By computing the average current by linear response in $A_{x}$ and defining the stiffness as $J_{s}=-\left\langle I_{x}\right\rangle / A_{x}$ one has

$$
\begin{gathered}
J_{s}=J_{d}-J_{p}, \\
J_{d}=\frac{J}{N}\left\langle\sum_{i} \cos \left(\theta_{i}-\theta_{i+\hat{x}}\right)\right\rangle, \\
J_{p}=\frac{J^{2}}{N T}\left\langle\left[\sum_{i} \sin \left(\theta_{i}-\theta_{i+\hat{x}}\right)\right]^{2}\right\rangle,
\end{gathered}
$$

where $T$ is the temperature. In Eq. (3) the second term is already linear in the external perturbation; thus, in linear response theory one can directly compute its average value leading to the diamagnetic contribution $J_{d}$ of Eq. (5), which coincides in this case with the average energy density along the $x$ direction. The first term in Eq. (3) defines instead the paramagnetic current, whose average value is connected to the current-current correlation function, leading to the paramagnetic term in Eq. (6). To estimate their contribution to the thermal suppression of the superfluid stiffness at low temperature we can approximate the phase difference between neighboring sites $\theta_{i+\mu}-\theta_{i}$ with a continuum gradient $\theta_{i+\mu}-\theta_{i} \approx \partial_{\mu} \theta\left(\mathbf{r}_{i}\right)$, where we set the lattice spacing $a=1$. By expanding also the cosine of Eq. (2) at leading order in the phase gradient we end up with a Gaussian model accounting only for spin-wave-like longitudinal phase fluctuations:

$$
H_{X Y} \simeq \frac{J}{2} \int d \mathbf{r}[\nabla \theta(\mathbf{r})]^{2} .
$$

The approximation (7) allows one to perform analytically the averages in Eqs. (5) and (6). For the diamagnetic term one immediately finds:

$$
J_{d} \simeq J-\frac{J}{2 N}\left\langle\int d \mathbf{r}\left[\partial_{x} \theta(\mathbf{r})\right]^{2}\right\rangle \simeq J-\frac{T}{2 d},
$$

where we used that fact that for the Gaussian model (7) $\left\langle\int d \mathbf{r}\left[\partial_{x} \theta(\mathbf{r})\right]^{2}\right\rangle=T / d J$, with $d=2$ being the space dimension. For the paramagnetic contribution, we expand the sine function in powers of the phase gradient. The correlation function (6) amounts then to the sum of processes where an odd number of phase modes (phasons) are excited by the electromagnetic field. However, in the clean case the first term, proportional to $\left\langle\int d \mathbf{r} d \mathbf{r}^{\prime} \partial_{x} \theta(\mathbf{r}) \partial_{x} \theta\left(\mathbf{r}^{\prime}\right)\right\rangle$, vanishes because of periodic boundary conditions. The next nonzero contribution is then a three-phason processes, which reads explicitly:

$$
\begin{aligned}
J_{p} & \simeq \frac{J^{2}}{T N}\left\langle\int d \mathbf{r} d \mathbf{r}^{\prime} \frac{1}{6}\left[\partial_{x} \theta(\mathbf{r})\right]^{3} \frac{1}{6}\left[\partial_{x} \theta\left(\mathbf{r}^{\prime}\right)\right]^{3}\right\rangle \\
& =J^{2} \frac{T^{2}}{6 d^{3}} .
\end{aligned}
$$

The analytical expressions (8) and (9) perfectly reproduce the low-temperature Monte Carlo simulations shown in Fig. 1. In particular the diamagnetic suppression (8) is the main source of temperature dependence of the stiffness up to $T \simeq 0.6$. As the temperature increases, two effects come into play. From one side higher-order terms in the phase gradient should be included, and more importantly, vortex-antivortex pairs start to form and unbind at the BKT transition, as shown in the bottom 


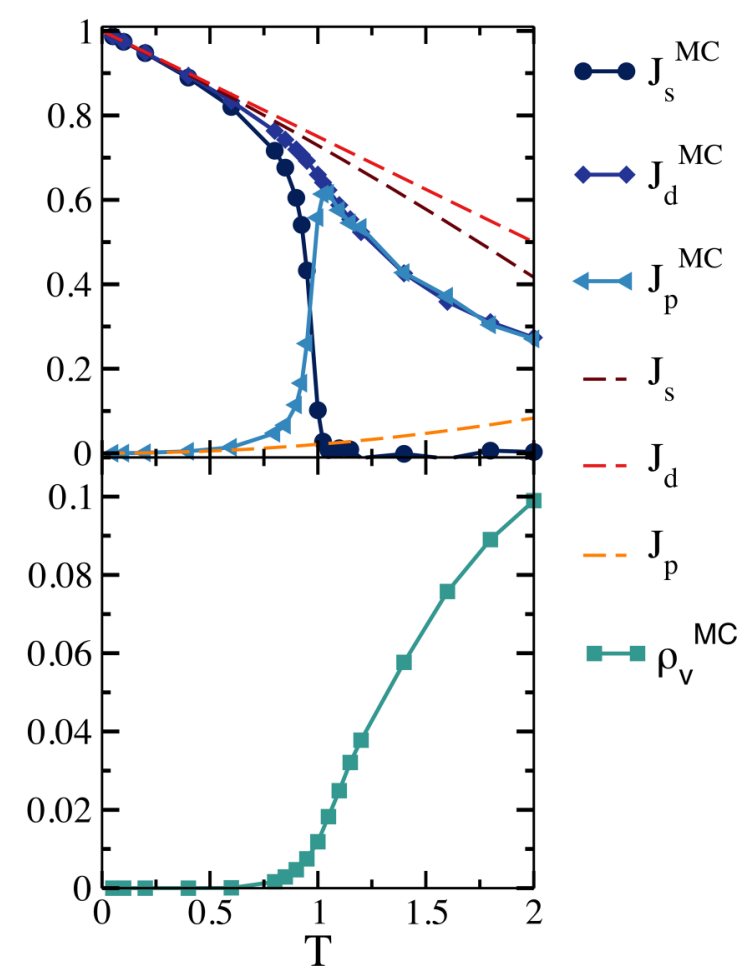

FIG. 1. Top: comparison between the Monte Carlo results and the low-temperature analytical expressions for the three response functions $J_{s}, J_{d}$, and $J_{p}$ based on Eqs. (8) and (9). Bottom: vortex density of the system as a function of the temperature. The linear size of the system simulated is $L=256$.

panel of Fig. 1. The latter effect appears predominantly in the paramagnetic contribution, which includes large-distance current correlations, while the diamagnetic one is predominantly local. As a consequence, $J_{p}$ sharply increases approaching $T_{c}$, causing the rapid downturn in the superfluid stiffness that is the signature of the universal BKT jump in simulations on a finite-size system. It is also worth noting that the dominance of the spin waves to suppress the low-temperature stiffness via the correction (8) of the diamagnetic term is also found in the more general SC (or spin) model where the SC amplitude (or spin length, set here to the unitary value) is not fixed, as investigated, e.g., in Ref. [54]. Indeed, at low temperature the amplitude fluctuations are thermally suppressed, making it possible only to thermally excite the low-energy longitudinal phase modes, responsible for the decrease in the diamagnetic term.

The general expressions (8) and (9) of the diamagnetic and paramagnetic temperature corrections in powers of the phase modes hold also for the quantum case. However, in this case the average value of the phase gradient should be computed using a quantum phase model, including the frequency dependence of the phase fluctuations [2-6]. The main consequence for the present discussion is that, below a crossover temperature $T_{c l}$, the classical (thermal) corrections to the superfluid stiffness discussed so far turn into quantum ones, leading finally to a $T=0$ value of the stiffness smaller than $J$ even for clean models. The exact form of the quantum corrections depends on the dynamics of the phase mode, which in turn is controlled by the presence of Coulomb interactions and dimensionality (see, e.g., [1,6]). In ordinary SC systems the quantum phase modes are pushed to the plasma energy scale, so that $T_{c l}$ can be larger than the critical temperature itself where quasiparticle excitations destroy the SC state, hindering the observation of phase-fluctuation effects on the superfluid stiffness. On the other hand, whenever the plasma energy scale is suppressed by correlations or disorder, $T_{c l}$ can be considerably reduced, making eventually classical phase-fluctuation corrections to the stiffness experimentally accessible. This possibility was discussed, for example, in the context of cuprate superconductors in Ref. [5]. There it was shown that the spectral function of the phase modes is modified by a residual absorption at low frequencies, induced by the sizable fraction of quasiparticle excitations present in a $d$-wave superconductor. This extra dissipation suppresses significantly the crossover temperature $T_{c l}$, which is given in this case by $T_{c l} \sim J / \bar{\sigma}$, where $J$ is the $T=0$ superfluid stiffness and $\bar{\sigma}$ is a dimensionless measure of the residual conductivity in the SC state. A similar mechanism can be at play also in conventional $s$-wave superconductors at strong disorder, which also show an extra subgap absorption (see Ref. [55] and references therein). The anomalous lineartemperature dependence of the superfluid stiffness measured in Ref. [11] has been indeed discussed within this context. For these reasons, the classical XY model studied in the present paper is not only interesting by itself but could also be relevant for applications to real systems.

\section{DISORDERED CASE}

Let us consider now the case where the local couplings $J_{i}^{\mu}$ of the model (1) are random variables extracted with a probability distribution $P(J)$. In full analogy with the clean case, the superfluid stiffness is defined via diamagnetic and paramagnetic terms, which are now also averaged over disorder configurations:

$$
\begin{gathered}
J_{d}=\frac{1}{N} \overline{\left\langle\sum_{i} J_{i}^{\mu} \cos \left(\theta_{i}-\theta_{i+\mu}\right)\right\rangle}, \\
J_{p}=\frac{1}{N T}\left\langle\overline{\left.\left[\sum_{i} J_{i}^{\mu} \sin \left(\theta_{i}-\theta_{i+\mu}\right)\right]^{2}\right\rangle} .\right.
\end{gathered}
$$

In the disordered case the $T=0$ value of the stiffness can be obtained by mapping the $\mathrm{SC}$ problem into the random-resistor network (RRN) one. The starting point is again a Gaussian approximation for the cosine term in Eq. (1), so that

$$
H_{X Y} \simeq-\sum_{i, \mu=\hat{x}, \hat{y}} J_{i}^{\mu}\left[1-\frac{1}{2}\left(\theta_{i}-\theta_{i+\mu}\right)^{2}\right] .
$$

The configuration of the phase variables can be obtained by the minimization of Eq. (12), giving a set of equations:

$$
\sum_{\mu= \pm \hat{x}, \hat{y}} J_{i}^{\mu}\left(\theta_{i}-\theta_{i+\mu}\right)=0
$$

In the absence of vortexlike excitations, finding the solutions $\theta_{i}$ of the system of Eq. (13) for a given set of $J_{i}^{\mu}$ values is equivalent to solving the Kirchhoff equations for the local 
voltages $V_{i}$ for an array of local random conductances $\sigma_{i}^{\mu}$. Indeed, by using the condition for the current conservation at each node between sites $i$ and $i+\mu$ one easily gets:

$$
\sum_{\mu= \pm \hat{x}, \hat{y}} i_{i}^{\mu}=\sum_{\mu= \pm \hat{x}, \hat{y}} \sigma_{i}^{\mu}\left(V_{i}-V_{i+\mu}\right)=0 .
$$

The comparison between Eqs. (13) and (14) establishes the equivalence between the local conductances $\sigma_{i}^{\mu}$ of the RRN and the local stiffnesses $J_{i}^{\mu}$ of the XY model. This also means that finding the global phase stiffness $J_{s}$ is equivalent to determining the global conductance $\sigma$ of the RRN problem. A possible solution for $\sigma$ was proposed long ago within the EMA scheme (see [52] and references therein). The basic idea is that the inhomogeneous system can be mapped into a homogeneous one characterized by an effective value $\tilde{\sigma}$ of the conductance such that, on average, the presence of a single disordered link with $\sigma_{i} \neq \tilde{\sigma}$ has vanishing effects on the current and voltage distributions of the system. The effective conductance $\tilde{\sigma}$ is then obtained as the solution of a selfconsistent equation, which for a cubic lattice in $d$ dimensions reads:

$$
\sum_{i} P_{i} \frac{\sigma_{i}-\tilde{\sigma}}{\sigma_{i}+(d-1) \tilde{\sigma}}=0
$$

where $P_{i}$ is the probability of the occurrence of each possible $\sigma_{i}$ value. Despite being based on a mean-field argument, Eq. (15) has been shown to capture very well the transport properties of disordered arrays of resistors. In the case, for example, of the diluted systems, where a given fraction $p$ of the resistors is set to zero, the percolation threshold $p=0.5$ obtained with Eq. (15) coincides with the results of numerical simulations [52].

In this paper we will provide a derivation of Eq. (15) for the superfluid stiffness based on resummed perturbation theory. Within our approach we will estimate not only the $T=0$ superfluid stiffness, but also its leading temperature dependence, giving an excellent description of Monte Carlo simulations. Moreover, we will derive the effect of disorder on the two separate diamagnetic and paramagnetic contributions, which could be relevant for the experiments. Indeed, thanks to the optical sum rule [28] one knows that the extra paramagnetic suppression of the stiffness induced by disorder transfers into a finite-frequency optical absorption. The quantum version of this mechanism was recently invoked to explain the extra subgap microwave absorption measured at low temperatures in strongly disordered superconductors (see, e.g., Refs. [30,31,55] and references therein). As mentioned below, the analogous classical effect investigated here could be relevant in real materials above the crossover temperature $T_{c l}$.

\section{A. Effective medium theory for the XY model at $T=0$}

As explained in Sec. II, at low temperature, where the topological phase excitations (vortices) still do not play a role, a continuum approximation for the model (1) allows one to easily describe the longitudinal phase fluctuations. In the disordered case we will follow the same strategy by also implementing the basic idea underlying the EMA. We then introduce a homogeneous effective stiffness $\tilde{J}$, and we will require that adding a single impurity with a local stiffness different from $\tilde{J}$ will have no overall effect on the system. Let us thus write the XY Hamiltonian as the sum of the two terms:

$$
H=H_{0}+H_{i}=\frac{\tilde{J}}{2} \int d \mathbf{r}[\nabla \theta(\mathbf{r})]^{2}+\frac{\delta J_{i}}{2}\left[\nabla \theta\left(\mathbf{r}_{\mathbf{i}}\right)\right]^{2},
$$

where we put $J_{i}=\tilde{J}+\delta J_{i}$. The second term of Eq. (16) can be seen as a perturbation with respect to $H_{0}$. We can then compute the self-energy correction $\Sigma_{i}$ to the bare Green's function $G_{0}=\left\langle\theta_{\mathbf{k}} \theta_{-\mathbf{k}}\right\rangle_{H_{0}}$ due to the presence of the impurity, so that the total Green's function $G(\mathbf{k})=\left\langle\theta_{\mathbf{k}} \theta_{-\mathbf{k}}\right\rangle_{H_{0}+H_{i}}$ reads:

$$
G(\mathbf{k})=G_{0}(\mathbf{k})+G_{0}(\mathbf{k}) \Sigma_{i}(\mathbf{k}) G_{0}(\mathbf{k}) .
$$

By expanding $e^{-\beta H_{i}}$ in a power series we can compute $G(\mathbf{k})$ as:

$$
G(\mathbf{k})=\left\langle\theta_{\mathbf{k}}\left\{\sum_{n=0}^{\infty} \frac{(\beta)^{n}}{n !}\left(-\frac{\delta J_{i}}{2}\right)^{n}\left[\nabla \theta\left(\mathbf{r}_{i}\right)\right]^{2 n}\right\} \theta_{-\mathbf{k}}\right\rangle_{H_{0}} .
$$

The first term $(n=0)$ is nothing but the bare Green's function $G_{0}(\mathbf{k})=T / \tilde{J} \mathbf{k}^{2}$. The remaining terms can be computed by means of Wick's theorem. For example, the second term $(n=1)$ reads

$$
\begin{aligned}
G_{1}(\mathbf{k}) & =\left\langle\theta_{\mathbf{k}}\left\{\frac{-\beta \delta J_{i}}{2}\left[\nabla \theta\left(\mathbf{r}_{i}\right)\right]^{2}\right\} \theta_{-\mathbf{k}}\right\rangle_{H_{0}} \\
& =\left\langle\theta_{\mathbf{k}}\left[\frac{-\beta \delta J_{i}}{2 N} \sum_{\mathbf{q}_{1}, \mathbf{q}_{2}} e^{i\left(\mathbf{q}_{1}+\mathbf{q}_{2}\right) \cdot \mathbf{r}_{\mathbf{i}}}\left(i \mathbf{q}_{\mathbf{1}}\right) \cdot\left(i \mathbf{q}_{\mathbf{2}}\right) \theta_{\mathbf{q}_{1}} \theta_{\mathbf{q}_{2}}\right] \theta_{-\mathbf{k}}\right\rangle_{H_{0}} \\
& =G_{0}(\mathbf{k})\left(-\frac{\delta J_{i}}{N} \frac{\mathbf{k}^{2}}{T}\right) G_{0}(\mathbf{k})=G_{0}(\mathbf{k}) \Sigma_{i}^{(1)}(\mathbf{k}) G_{0}(\mathbf{k}),
\end{aligned}
$$

where the prefactor $1 / 2$ canceled out with the diagram multiplicity. The factor $1 / N$, due to the fact that we are considering one single impurity, will be omitted in what follows. Indeed, as soon as one considers the original model with $N$ noninteracting possible impurities, the sum over all impurities cancels out the $N$ prefactor. For higher-order terms this procedure implements the usual $T$-matrix approximation [53], where only noncrossing diagrams with multiple-scattering events by different impurities are included. It is then easy to verify that the $n$th term of the expansion reads:

$$
\begin{aligned}
G_{n}(\mathbf{k}) & =G_{0}(\mathbf{k})\left[(-1)^{n} \frac{\mathbf{k}^{2}}{T}\left(\frac{\delta J_{i}}{d \tilde{J}}\right)^{n} d \tilde{J}\right] G_{0}(\mathbf{k}) \\
& =G_{0}(\mathbf{k}) \Sigma_{i}^{(n)}(\mathbf{k}) G_{0}(\mathbf{k}) .
\end{aligned}
$$

Therefore, we can write the full local single-impurity self-energy as:

$$
\Sigma_{i}(\mathbf{k})=\sum_{n=1}^{\infty} \Sigma_{i}^{(n)}(\mathbf{k})=\sum_{n=1}^{\infty}(-1)^{n} \frac{\mathbf{k}^{2}}{T}\left(\frac{\delta J_{i}}{d \tilde{J}}\right)^{n} d \tilde{J},
$$

whose diagrammatic representation is shown in Fig. 2. The irrelevance of the single-impurity perturbation on the physical responses of the system translates, in this approach, to the request of a vanishing local self-energy at all orders in the 


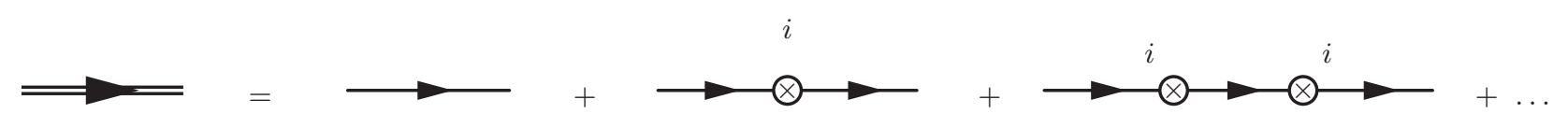

FIG. 2. Diagrammatic representation of the local self-energy corrections to the bare Green's function at $T=0$. Here the solid line represents the bare Green's function $G_{0}$, the double line is the dressed one $G$, and each cross accounts for a single-impurity scattering, contributing with a factor proportional to $\delta J_{i}$.

perturbation. This, in turn, is satisfied if:

$$
\sum_{i} P_{i} \sum_{n=1}^{\infty}(-1)^{n}\left(\frac{\delta J_{i}}{d \tilde{J}}\right)^{n}=0
$$

where we also included the average over all the possible values of $\delta J_{i}$, extracted from the probability distribution $P_{i} \equiv$ $P\left(J_{i}\right)$. Since $\delta J_{i}=\tilde{J}-J_{i}$, we can rewrite Eq. (22) as:

$$
\sum_{i} P_{i}\left[\sum_{n=0}^{\infty}(-1)^{n}\left(\frac{\tilde{J}-J_{i}}{d \tilde{J}}\right)^{n}-1\right]=0,
$$

which, after simple algebra, is equivalent to:

$$
\sum_{i} P_{i}\left[\frac{J_{i}-\tilde{J}}{J_{i}+(d-1) \tilde{J}}\right]=0
$$

By direct comparison with (15) we immediately see that $\tilde{J}$ satisfies the same equation of the effective conductance of the RRN model within EMA. While this was expected on the basis of the formal analogy between the two problems encoded in Eqs. (13) and (14), our derivation of the equivalence between the EMA equation and the perturbative expansion in the disorder potential brings interesting consequences. Following the same procedure, we can compute separately the zero-temperature values of the diamagnetic $J_{d}(T=0)$ and paramagnetic $J_{p}(T=0)$ contributions in order to understand how the diagrammatic expansion in $H_{i}$ affects the clean-limit results. We start by gradient expansion of Eqs. (10) and (11). For the diamagnetic contribution the first-order correction in the cosine expansion already gives a finite- $T$ correction, in full analogy with Eq. (8) above, so we simply have:

$$
J_{d}(T=0) \simeq \frac{1}{N} \overline{\left\langle\int d \mathbf{r} J(\mathbf{r})\right\rangle_{H_{0}+H_{i}}}=\bar{J},
$$

showing that at all orders in the perturbing potential the zerotemperature diamagnetic response coincides with the mean value $\bar{J}$ of the couplings. For the paramagnetic term disorder makes different from zero the single-phason process discussed in the previous section, which then reads:

$$
J_{p} \simeq \frac{1}{N T} \overline{\left\langle\int d \mathbf{r} J(\mathbf{r})\left[\nabla_{x} \theta(\mathbf{r})\right] \int d \mathbf{r}^{\prime} J\left(\mathbf{r}^{\prime}\right)\left[\nabla_{x} \theta\left(\mathbf{r}^{\prime}\right)\right]\right\rangle_{H_{0}+H_{i}}} .
$$

By using the facts that $J(\mathbf{r})=\tilde{J}$ everywhere except at the single-impurity site where $J_{i}=\tilde{J}+\delta J_{i}$ and that the contribution proportional to the homogeneous stiffness $\tilde{J}$ vanishes because of periodic boundary conditions, we immediately get:

$$
\left.J_{p} \simeq \frac{1}{N T} \overline{\left\langle\left\{\left(\delta J_{i}\right)^{2}\left[\nabla_{x} \theta\left(\mathbf{r}_{\mathbf{i}}\right)\right]^{2}\right\}\right\rangle}\right\rangle_{H_{0}+H_{i}}
$$

Now, by omitting as before one overall prefactor $1 / N$, we proceed with the perturbation expansion in $H_{i}$. The first term $(n=0)$ is simply:

$$
\begin{aligned}
J_{p_{(n=0)}} & =\frac{\left(\delta J_{i}\right)^{2}}{N T}\left\langle\sum_{\mathbf{q}_{1}, \mathbf{q}_{2}} e^{i\left(\mathbf{q}_{1}+\mathbf{q}_{2}\right) \cdot \mathbf{r}_{i}}\left(i \mathbf{q}_{1}^{x}\right)\left(i \mathbf{q}_{2}^{x}\right) \theta_{\mathbf{q}_{1}} \theta_{\mathbf{q}_{2}}\right\rangle_{H_{0}} \\
& =\frac{\left(\delta J_{i}\right)^{2}}{d \tilde{J}} .
\end{aligned}
$$

The second term ( $n=1)$ will be, instead,

$$
\begin{aligned}
J_{p_{(n=1)}}= & \frac{-\left(\delta J_{i}\right)^{3}}{2 N^{2} T}\left\langle\sum_{\mathbf{q}_{1}, \mathbf{q}_{2}} \sum_{\mathbf{k}_{1}, \mathbf{k}_{2}} e^{i\left(\mathbf{q}_{1}+\mathbf{q}_{2}+\mathbf{k}_{1}+\mathbf{k}_{2}\right) \cdot \mathbf{r}_{i}} .\right. \\
& \left.\times \mathbf{q}_{1}^{x} \mathbf{q}_{2}^{x} \mathbf{k}_{1} \mathbf{k}_{2} \theta_{\mathbf{q}_{1}} \theta_{\mathbf{q}_{2}} \theta_{\mathbf{k}_{1}} \theta_{\mathbf{k}_{2}}\right\rangle_{H_{0}},
\end{aligned}
$$

which after the contractions reads:

$$
J_{p_{(n=1)}}=-\frac{\left(\delta J_{i}\right)^{3}}{d^{2} \tilde{J}^{2}} .
$$

By proceeding analogously at all orders in the perturbative expansion one can write the zero-temperature value of the paramagnetic term as:

$$
\begin{aligned}
J_{p}(T=0) & =\sum_{i} P_{i}\left[d \tilde{J} \sum_{n=2}^{\infty}(-1)^{n}\left(\frac{\delta J_{i}}{d \tilde{J}}\right)^{n}\right] \\
& =\sum_{i} P_{i}\left(d \tilde{J}\left\{\left[\sum_{n=1}^{\infty}(-1)^{n}\left(\frac{\delta J_{i}}{d \tilde{J}}\right)^{n}\right]-\left(-\frac{\delta J_{i}}{d \tilde{J}}\right)\right\}\right) \\
& =\sum_{i} P_{i}\left[d \tilde{J} \frac{\delta J_{i}}{d \tilde{J}}\right]=\sum_{i} P_{i}\left(J_{i}-\tilde{J}\right)=\bar{J}-\tilde{J}, \quad(31)
\end{aligned}
$$

where, in the last step, we used the result of Eq. (22). Equations (25) and (31) clearly satisfy the general relation (4), as expected. On the other hand, their separate evaluation helps us understand the different roles of disorder in the two terms. Indeed, while the diamagnetic term (25) is a measure of the average disorder distribution, the paramagnetic one is a measure of its variance, as one immediately sees from the leading correction (28). This results will help us explain the difference between Gaussian and diluted disorder in Sec. IV.

\section{B. Effective medium theory for the XY model up to linear terms in $T$}

Let us now extend the $T=0$ results in order to estimate the leading temperature corrections to $J_{s}, J_{d}$, and $J_{p}$. As for the $T=0$ case, we will account here only for the longitudinal spin fluctuations, since the topological excitations are strongly suppressed in the low-temperature regime considered. To this 


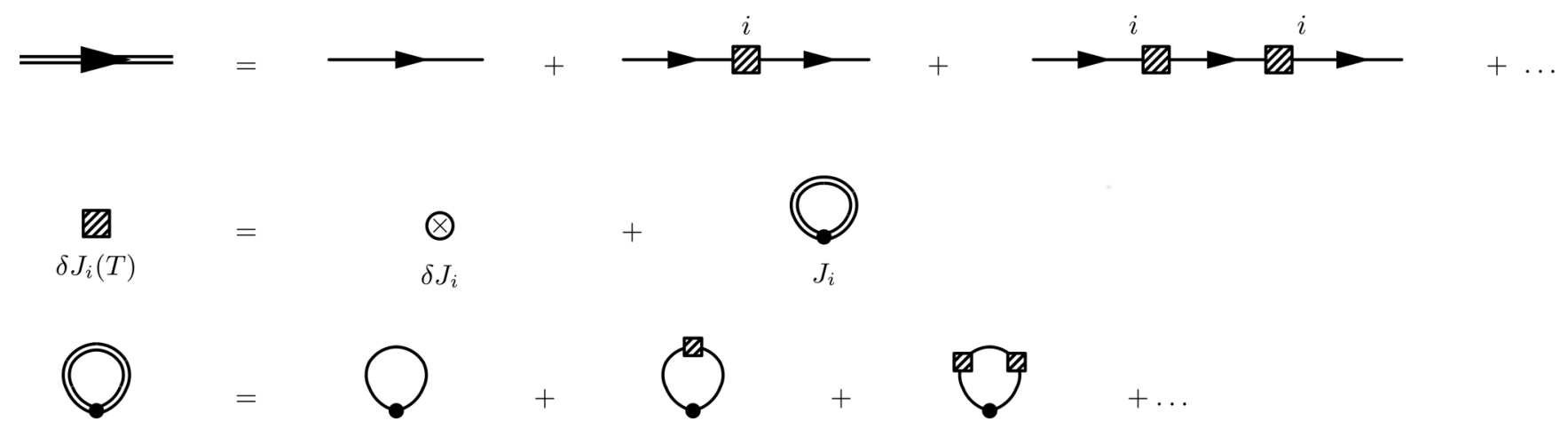

FIG. 3. Diagrammatic representation of the local self-energy corrections to the bare Green's function up to the linear terms in temperature. In the diagrammatic expansion, each arrow stands for a bare Green's function, while the hatched square accounts for two different impurityscattering contributions. As shown in the second line, indeed, it corresponds to the sum of the zero-temperature correction, proportional to $\delta J_{i}$, and the linear temperature one, proportional to $J_{i}$, arising from the four-leg vertex in $H_{i}^{(4)}$. The closed loop appearing in the second line accounts self-consistently for all the local self-energy corrections, as shown in the last line. As a matter of fact, however, since we are considering the self-energy corrections up to the linear terms in temperature, in our calculation we will consider the closed loop corrected by only the zero-temperature contribution; that is, we will replace the hatched square with the bare circle $\delta J_{i}$.

aim we need to consider that each $G_{0}$ carries a power of $T$. By power counting it, it is then clear that at finite $T$ it is crucial to retain in the disorder Hamiltonian $H_{i}$ also the quartic term in the expansion of the cosine, since each additional $(\nabla \theta)^{2}$ term will contribute with an extra power of $T / J$ in the perturbative expansion:

$$
H_{i}=H_{i}^{(2)}+H_{i}^{(4)}=\frac{\delta J_{i}}{2}\left[\nabla \theta\left(\mathbf{r}_{i}\right)\right]^{2}-\frac{J_{i}}{4 !} \sum_{\mu=\hat{x}, \hat{y}}\left[\nabla_{\mu} \theta\left(\mathbf{r}_{\mathbf{i}}\right)\right]^{4} .
$$

From the diagrammatic point of view, the quartic $H_{i}^{(4)}$ term in $\nabla \theta\left(\mathbf{r}_{\mathbf{i}}\right)$ introduces a four-leg vertex in the phase field, whose combination with the two-leg one in $H_{i}^{(2)}$ complicates the calculation of the Green's function, which will be carried out along the same lines as Eq. (18). The easiest way to handle this problem is to follow the same logic as in the zero-temperature case, as summarized in Fig. 3. When computing the full Green's function (double line) we sum up only noncrossing diagrams with multiple scattering by a single-impurity site. However, we will replace $\delta J_{i}$ (crossed circle) with its finitetemperature value $\delta J_{i}(T)$ :

$$
\delta J_{i} \rightarrow \delta J_{i}(T)
$$

In this way, $\delta J_{i}(T)$ accounts for scattering events described both by the $H_{i}^{(2)}$ two-leg vertex insertions and the $H_{i}^{(4)}$ fourleg vertex insertion, which generates a loop diagram with the full Green's function, as shown in Fig. 3. The first order of the new local self-energy is then obtained with the second line of Fig. 3. The first term is the zero-temperature contribution, already given in Eq. (19):

$$
G_{(n=1)}(\mathbf{k}, O(1))=G_{0}(\mathbf{k})\left(-\delta J_{i} \frac{\mathbf{k}^{2}}{T}\right) G_{0}(\mathbf{k}) .
$$

The second term arises from the four-leg vertex, and it corresponds to a loop diagram, as shown in the second line of
Fig. 3:

$$
\begin{aligned}
& G_{(n=1)}(\mathbf{k}, O(T)) \\
& \quad=\left\langle\theta_{\mathbf{k}}\left\{\frac{\beta}{4 !} J_{i}\left[\nabla_{x} \theta\left(\mathbf{r}_{i}\right)\right]^{4}+\left[\nabla_{y} \theta\left(\mathbf{r}_{i}\right)\right]^{4}\right\} \theta_{-\mathbf{k}}\right\rangle_{H_{0}},
\end{aligned}
$$

where $J_{i}=\tilde{J}+\delta J_{i}$. We can then rewrite Eq. (35) in momentum space as:

$$
\begin{aligned}
G_{(n=1)}(\mathbf{k}, O(T))= & 2\left\langle\theta _ { \mathbf { k } } \left[\frac{\beta J_{i}}{4 !} \sum_{\mathbf{q}_{1}, \mathbf{q}_{2}, \mathbf{q}_{3}, \mathbf{q}_{4}} e^{i\left(\mathbf{q}_{1}+\mathbf{q}_{2}+\mathbf{q}_{3}+\mathbf{q}_{4}\right) \cdot \mathbf{r}_{i}}\right.\right. \\
& \left.\left.\times \mathbf{q}_{1}^{x} \mathbf{q}_{2}^{x} \mathbf{q}_{3}^{x} \mathbf{q}_{4}^{x} \theta_{\mathbf{q}_{1}} \theta_{\mathbf{q}_{2}} \theta_{\mathbf{q}_{3}} \theta_{\mathbf{q}_{4}}\right] \theta_{-\mathbf{k}}\right\rangle_{H_{0}},
\end{aligned}
$$

where the prefactor 2 accounts for the gradient along $y$ in Eq. (35). We then proceed with Wick's theorem, ending up with:

$$
\begin{aligned}
& G_{(n=1)}(\mathbf{k}, O(T)) \\
& \quad=G_{0}(\mathbf{k})\left[\beta J_{i} \frac{\mathbf{k}^{2}}{2 d} \sum_{\mathbf{q}} \mathbf{q}^{2}\left\langle\theta_{\mathbf{q}} \theta-\mathbf{q}\right\rangle_{H_{0}}\right] G_{0}(\mathbf{k}) .
\end{aligned}
$$

The quantity in square brackets in the above equation defines the Green's function in the loop, which at first order coincides with $G_{0}$. However, higher-order terms in the expansion of $H_{i}$ lead to the dressing of this loop by all the possible singleimpurity scattering processes, as shown by the last line of Fig. 3. The easiest way to sum them up is to replace the average over $H_{0}$ in Eq. (37) with an average over $H_{0}+H_{i}$. In addition, since this is already a finite-temperature correction, we can restrict ourselves to the terms generated by $H_{i}^{(2)}$, as 
done for the $T=0$ case. We then have:

$$
\begin{aligned}
& G_{(n=1)}(\mathbf{k}, O(T)) \\
& \quad=G_{0}(\mathbf{k})\left[\beta J_{i} \frac{\mathbf{k}^{2}}{2 d} \sum_{\mathbf{q}} \mathbf{q}^{2}\left\langle\theta_{\mathbf{q}} \theta-\mathbf{q}\right\rangle_{H_{0}+H_{i}^{(2)}}\right] G_{0}(\mathbf{k}) \\
& \quad=G_{0}(\mathbf{k})\left\{\beta J_{i} \frac{\mathbf{k}^{2}}{2 d} \sum_{\mathbf{q}} \mathbf{q}^{2} G_{0}(\mathbf{q})\left[\sum_{m=0}^{\infty}(-1)^{m}\left(\frac{\delta J_{i}}{d \tilde{J}}\right)^{m}\right]\right\} G_{0}(\mathbf{k}) .
\end{aligned}
$$

Solving then the geometric series in the last line, we end up with:

$$
G_{(n=1)}(\mathbf{k}, O(T))=G_{0}(\mathbf{k})\left[\frac{\mathbf{k}^{2}}{2} \frac{J_{i}}{d \tilde{J}+\delta J_{i}}\right] G_{0}(\mathbf{k}) .
$$

Finally, putting together Eqs. (34) and (39), we obtain the explicit expression for the single-scattering diagram on the upper line of Fig. 3:

$$
\begin{aligned}
G_{(n=1)}(\mathbf{k}) & =G_{0}(\mathbf{k})\left[-\frac{\mathbf{k}^{2}}{T}\left(\delta J_{i}-\frac{T}{2} \frac{J_{i}}{(d-1) \tilde{J}+J_{i}}\right)\right] G_{0}(\mathbf{k}) \\
& =G_{0}(\mathbf{k})\left[-\frac{\mathbf{k}^{2}}{T} \delta J_{i}(T)\right] G_{0}(\mathbf{k}),
\end{aligned}
$$

which is the equivalent of Eq. (19) up to linear terms in temperature. Thus, in perfect analogy with Eq. (22), the new EMA equation reads:

$$
\sum_{i} P_{i} \sum_{n=1}^{\infty}(-1)^{n}\left(\frac{\delta J_{i}(T)}{d \tilde{J}(T)}\right)^{n}=\sum_{i} P_{i} \frac{-\delta J_{i}(T)}{d \tilde{J}(T)+\delta J_{i}(T)}=0,
$$

where $\delta J_{i}(T)$ is given by:

$$
\delta J_{i}(T)=\delta J_{i}-\frac{T}{2} \frac{J_{i}}{(d-1) \tilde{J}(T)+J_{i}} .
$$

The same strategy can now be used to compute the finitetemperature corrections to the diamagnetic and paramagnetic terms. For the diamagnetic response the leading dependence on temperature is given by the first term in the cosine expansion of Eq. (10), so that:

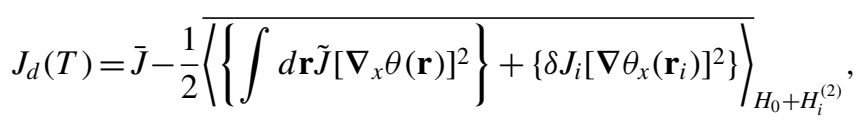

where we used again only the two-leg vertex of the impurity Hamiltonian since this term is already of $O(T)$, as already seen in the clean case (8). By means of the same formalism used so far, it is easy to verify that the first temperature correction reads:

$$
\begin{aligned}
& -\frac{1}{2} \overline{\left\lfloor d \mathbf{r} \tilde{J}\left[\nabla_{x} \theta(\mathbf{r})\right]^{2}\right\rangle_{H_{0}+H_{i}^{(2)}}} \\
& =-\frac{T}{2 d}\left[1+\sum_{i} P_{i} \sum_{n=1}^{\infty}(-1)^{n}\left(\frac{\delta J_{i}}{d \tilde{J}}\right)^{n}\right]=-\frac{T}{2 d},
\end{aligned}
$$

where in the last step we used Eq. (22), which accounts for the vanishing of the local self-energy. For the same reason the second temperature correction also vanishes:

$$
\begin{aligned}
& -\frac{1}{2} \overline{\left\langle\delta J_{i}\left[\nabla_{x} \theta\left(\mathbf{r}_{i}\right)\right]^{2}\right\rangle_{H_{0}+H_{i}^{(2)}}} \\
& =\frac{T}{2}\left[\sum_{i} P_{i} \sum_{n=1}^{\infty}(-1)^{n}\left(\frac{\delta J_{i}}{d \tilde{J}}\right)^{n}\right]=0 .
\end{aligned}
$$

Hence, at all orders in the perturbative expansion, the diamagnetic response function, up to the linear terms in temperature, depends only on the mean value of the random couplings and on the dimension of the system:

$$
J_{d}(T)=\bar{J}-\frac{T}{2 d}
$$

showing a very remarkable universality with respect to the random coupling distribution itself. Finally, the temperature dependence of the paramagnetic term $J_{p}(T)=J_{d}(T)-\tilde{J}(T)$ can be obtained by combining the results (41) and (46) for $\tilde{J}(T)$ and for $J_{d}(T)$, respectively.

\section{COMPARISON WITH THE MONTE CARLO RESULTS}

In this section, we will compare the analytical results, previously derived, with the numerical solutions obtained by means of Monte Carlo simulations of the classical XY model in the presence of random, spatially uncorrelated couplings $J_{i}^{\mu}$. The Monte Carlo simulations have been performed on systems with linear size $L=128$ and periodic boundary conditions. Each Monte Carlo step consists of five Metropolis spin flips of the whole lattice, needed to probe the correct canonical distribution of the system, followed by ten overrelaxation sweeps of all the spins, which help the thermalization. For each temperature we perform 5000 Monte Carlo steps, and we compute a given quantity averaged over the last 3000 steps, discarding thus the transient regime which occurs in the first 2000 steps. Furthermore, the thermalization at low temperatures is speeded up by a temperature annealing procedure. Finally, the average over disorder is done on 15 independent configurations for each disorder level considered. Where not shown, the error bars are smaller than the point size.

We will consider two different disorder distributions for the couplings $J_{i}^{\mu}$, showing that in both cases the EMA equations previously obtained are in very good agreement with the numerical results. We first consider the case of a Gaussian distribution:

$$
P\left(J_{i}^{\mu}\right)=\frac{1}{\sqrt{2 \pi \sigma}} \exp \left[-\frac{\left(J_{i}^{\mu}-J\right)^{2}}{2 \sigma^{2}}\right]
$$

where $J$ is set equal to 1 and the standard deviation $\sigma$ measures the disorder strength. We also consider the additional constraint of $J_{i}^{\mu} \geqslant 0$ to prevent the presence of antiferromagnetic couplings. The zero-temperature value of the stiffness can be easily obtained by means of the explicit expressions for the diamagnetic and paramagnetic contributions derived in Sec. III A. Indeed, by using Eqs. (25) and (31) we can easily 
estimate

$$
\begin{gathered}
J_{d}(T=0)=\bar{J}, \\
J_{p}(T=0)=\frac{\sigma_{J}^{2}}{d \bar{J}}, \\
\tilde{J}(T=0)=\bar{J}\left[1-\frac{\sigma_{J}^{2}}{d \bar{J}^{2}}\right],
\end{gathered}
$$

where for the paramagnetic term we just retained the leading term in $\delta J_{i}$, as given by Eq. (28).

Equation (50) could also be obtained [28] by directly solving the EMA equation (24) at leading order in $\delta J_{i}$. At finite temperature, we will indeed follow this procedure, starting from the self-consistency equation (41). Since for the Gaussian distribution all the odd momenta are, on average, zero, it is convenient to express $J_{i}=\bar{J}+\Delta J_{i}$. We can then rewrite Eq. (41) as:

$$
\sum_{i} P_{i}\left\{\frac{\bar{J}+\Delta J_{i}-\tilde{J}-\frac{T}{2} \frac{\bar{J}+\Delta J_{i}}{(d-1) \tilde{J}+\bar{J}+\Delta J_{i}}}{d \tilde{J}+\bar{J}+\Delta J_{i}-\tilde{J}-\frac{T}{2} \frac{\bar{J}+\Delta J_{i}}{(d-1) \tilde{J}+\bar{J}+\Delta J_{i}}}\right\}=0 .
$$

By retaining all the terms of orders $O(T)$ and $O\left(\left(\Delta J_{i}\right)^{2}\right)$, after simple algebra we obtain:

$$
\tilde{J}\left(1+\frac{T}{2 d^{2} \bar{J}}\right) \simeq \bar{J}\left(1+\frac{T}{2 d^{2} \bar{J}}\right)-\frac{\sigma_{J}^{2}}{d \tilde{J}}-\frac{T}{2 d}
$$

so that the effective stiffness reads:

$$
\tilde{J} \simeq \bar{J}\left[1-\frac{\sigma_{J}^{2}}{d \bar{J}^{2}}\right]-\frac{T}{2 d} .
$$

Finally, by using the general result (46) for the linear temperature dependence of the diamagnetic term, we can derive separate expressions for both $J_{d}(T)$ and $J_{p}(T)$ :

$$
\begin{gathered}
J_{d}(T) \simeq \bar{J}-\frac{T}{2 d}, \\
J_{p}(T) \simeq \frac{\sigma_{J}^{2}}{d \bar{J}} .
\end{gathered}
$$

Equations (53)-(55) can now be compared with the numerical simulations. In Fig. 4, we can see that for all values of $\sigma$, the analytical results fit very well the Monte Carlo data. Notice that for the case of $\sigma=0.6$ the truncation at $J_{i}^{\mu} \geqslant 0$ shifts the mean value of the couplings to $\bar{J}_{\sigma=0.6} \simeq 1.06 \mathrm{~J}>J$. Nevertheless, by using this value in Eqs. (53)-(55), we can perfectly reproduce the numerical results.

The second kind of spatially uncorrelated disorder investigated is that of random diluted couplings, whose probability distribution reads:

$$
P\left(J_{i}^{\mu}\right)=p \delta\left(J_{i}^{\mu}\right)+(1-p) \delta\left(J_{i}^{\mu}-J\right),
$$

where $\delta(x)$ is the Dirac delta function and the dilution parameter $p$ will be the measure of the disorder strength. Also in this case we will set $J=1$ in the simulations. In this case, we
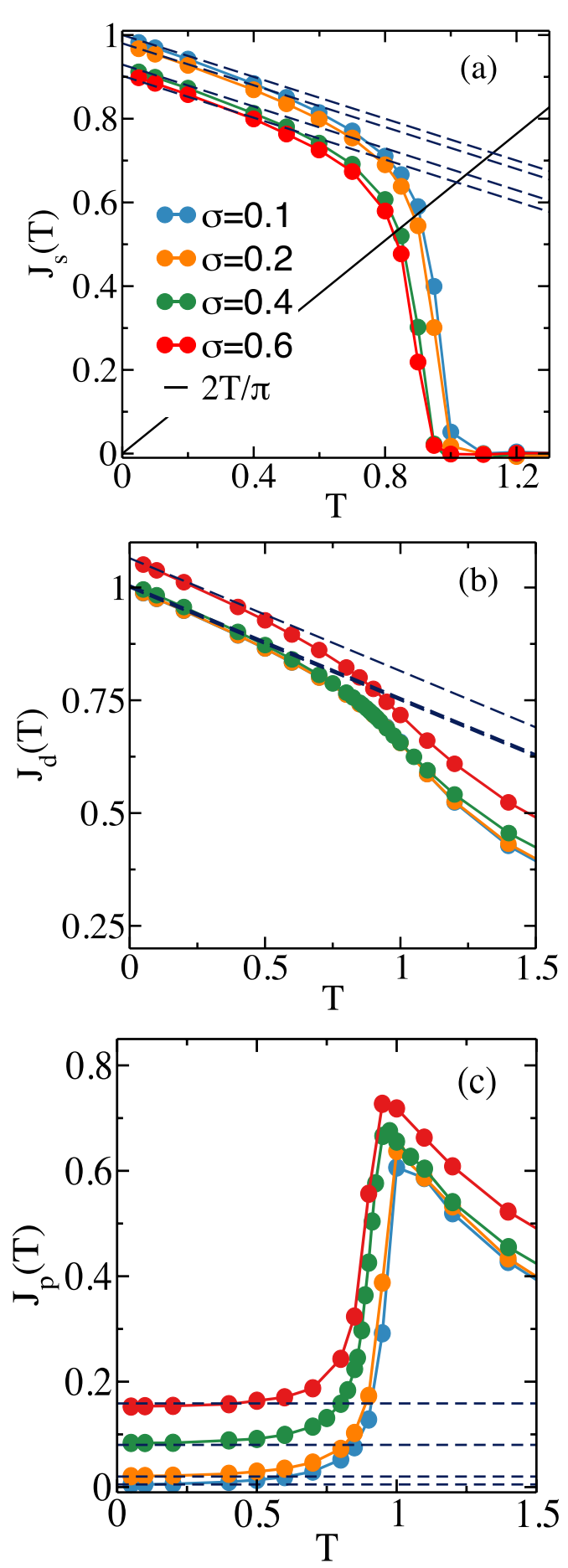

FIG. 4. Monte Carlo results on a disordered XY model with Gaussian distributed random couplings. The three response functions are plotted: (a) $J_{s}$, (b) $J_{d}$, and (c) $J_{p}$ as a function of the temperature. The dashed lines correspond to the analytical results (53)(55) obtained with the EMA and reproduce perfectly the numerical simulations.

cannot expect that the leading expansion in $\delta J_{i}$ holds since for the diluted model the variance of the distribution has the same order as its average value. However, in this case the EMA equation can be explicitly solved. By considering directly the 
finite-temperature case Eq. (41) reads:

$$
\begin{aligned}
& \sum_{i} P_{i} \frac{-\delta J_{i}(T)}{d \tilde{J}+\delta J_{i}(T)} \\
& \quad=(1-p) \frac{\tilde{J}-J+\frac{T}{2} \frac{1}{(d-1) \tilde{J}+J}}{(d-1) \tilde{J}+J-\frac{T}{2} \frac{1}{(d-1) \tilde{J}+J}}+p=0,
\end{aligned}
$$

which, after some math, leads to:

$$
\begin{aligned}
\tilde{J} & =J(1-2 p)\left(1-\frac{T}{2(J+\tilde{J})}\right) \\
& \simeq J(1-2 p)\left(1-\frac{T}{4 J(1-p)}\right),
\end{aligned}
$$

where in the last step we replaced $\tilde{J}$ in the denominator with its zero-temperature value. Using again Eq. (46) for the diamagnetic term, with $\bar{J}=J(1-p)$, we have the final expressions for all three contributions:

$$
\begin{gathered}
J_{s}=J(1-2 p)\left[1-\frac{T}{4 J(1-p)}\right], \\
J_{d}=J(1-p)-\frac{T}{4} \\
J_{p}=J p-\frac{T}{4} \frac{p}{1-p} .
\end{gathered}
$$

In Fig. 5 we show the Monte Carlo results for the three separate contributions. As one can see, the zero-temperature value of $J_{p}$ matches reasonably well the analytical estimate for $p=0.1$, while it slightly increases for larger disorder. Nonetheless, once accounting for this small deviation, by replacing $J p$ in Eq. (61) with the numerical value of $J_{p}(T=$ 0 ), the temperature dependence of the paramagnetic response function still follows the analytical result, as one can see by looking at the dashed lines in Fig. 5. In contrast, $J_{d}(T)$ follows closely the analytical estimate (60) up to the largest disorder level. This suggests that, in analogy with what was mentioned before for the thermal effects due to vortices, thermal effects due to longitudinal phase fluctuations also affect the paramagnetic contribution more strongly than the diamagnetic one. This is presumably due to the long-range character of the correlations probed by the current-current response function, compared to the local nature of the diamagnetic response. As a consequence, while for the Gaussian distribution the leading temperature effects due to longitudinal phase fluctuations can be solely ascribed to the diamagnetic correction, for the diluted model the paramagnetic response is responsible for a substantial flattening of the thermal suppression of the superfluid stiffness, causing in turn an increase in the $T_{c} / J_{s}(0)$ ratio.

\section{CONCLUSIONS}

In summary, we analyzed the effect of spatially uncorrelated random disorder on the low-temperature behavior of the superfluid stiffness of the 2D classical XY model. We employed a perturbative expansion in the disorder potential that is analogous to the usual $T$-matrix scheme including only noncrossing diagrams. We found that this approach allows one to derive a self-consistency equation for the global stiffness that
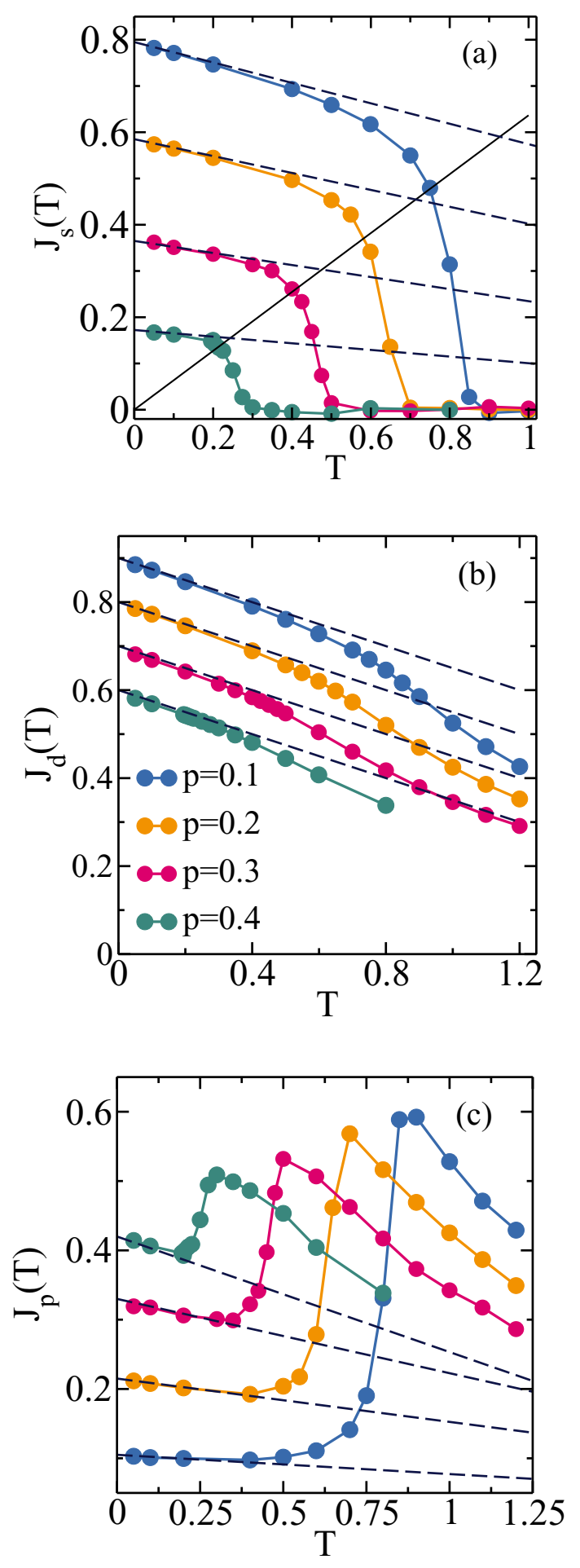

FIG. 5. Monte Carlo results for a disordered XY model with random diluted couplings. The three response functions are plotted: (a) $J_{s}$, (b) $J_{d}$, and (c) $J_{p}$ as a function of the temperature. The dashed lines correspond to the analytical results (59)-(61) obtained with the EMA. Even close to the percolation threshold at $p=0.5$, above which no superfluidity is possible, the analytical formulas fit very well the numerical data.

is fully equivalent to the usual EMA, usually discussed within the context of the RRN model [52]. This result leads to two interesting consequences. First, it allows one to incorporate 
also the finite-temperature corrections. This leads to the modified EMA equation (41) for the stiffness, which properly describes the thermal suppression of the stiffness due to longitudinal phase modes in the presence of disorder. Second, it allows one to compute separately the diamagnetic and paramagnetic contributions to the stiffness. This is in turn crucial information in order to establish the fraction of the total SC spectral weight which is transferred, thanks to the optical sum rule [28], to the finite-frequency absorption. These analytical findings offer an excellent description of the Monte Carlo results for both the Gaussian and diluted models of disorder. In the latter case the only discrepancy is a slightly larger paramagnetic suppression of the stiffness at $T=0$ for large disorder, which can presumably be ascribed to emerging space-correlation effects, neglected by the $T$-matrix scheme. However, it is interesting to note that the resulting temperature suppression of the stiffness turns out to be weaker in the diluted model with respect to the homogeneous case. This result has to be contrasted with the recent Monte Carlo simulations [42] done with a granular space-correlated model of disorder. In this case the stronger thermal suppression of the stiffness with respect to the homogeneous case had been attributed to a low-temperature proliferation of vortex-antivortex pairs in the bad SC regions. These opposite trends suggest that disorder not only affects the temperature scales where longitudinal (spin-wave like) and transverse (vortex-like) phase fluctuations become visible but can also profoundly change their interplay. Understanding how this interplay evolves as a function of disorder and how it can be relevant for 2D SC systems is an open question for future work.

\section{ACKNOWLEDGMENTS}

This work was supported by Italian MAECI under the Italian-India collaborative Project SUPERTOPPGR04879. We acknowledge the cost action Nanocohybri CA16218.
[1] R. Fazio and H. van der Zant, Phys. Rep. 355, 235 (2001).

[2] S. De Palo, C. Castellani, C. Di Castro, and B. K. Chakraverty, Phys. Rev. B 60, 564 (1999).

[3] A. Paramekanti, M. Randeria, T. V. Ramakrishnan, and S. S. Mandal, Phys. Rev. B 62, 6786 (2000).

[4] S. G. Sharapov, H. Beck, and V. M. Loktev, Phys. Rev. B 64, 134519 (2001).

[5] L. Benfatto, S. Caprara, C. Castellani, A. Paramekanti, and M. Randeria, Phys. Rev. B 63, 174513 (2001).

[6] L. Benfatto, A. Toschi, and S. Caprara, Phys. Rev. B 69, 184510 (2004).

[7] V. L. Berezinskii, Sov. Phys. JETP 34, 610 (1972).

[8] J. M. Kosterlitz and D. J. Thouless, J. Phys. C 6, 1181 (1973).

[9] J. M. Kosterlitz, J. Phys. C 7, 1046 (1974).

[10] B. Sacépé, C. Chapelier, T. I. Baturina, V. M. Vinokur, M. R. Baklanov, and M. Sanquer, Nat. Commun. 1, 140 (2010).

[11] M. Mondal, A. Kamlapure, M. Chand, G. Saraswat, S. Kumar, J. Jesudasan, L. Benfatto, V. Tripathi, and P. Raychaudhuri, Phys. Rev. Lett. 106, 047001 (2011).

[12] M. Chand, G. Saraswat, A. Kamlapure, M. Mondal, S. Kumar, J. Jesudasan, V. Bagwe, L. Benfatto, V. Tripathi, and P. Raychaudhuri, Phys. Rev. B 85, 014508 (2012).

[13] D. Sherman, G. Kopnov, D. Shahar, and A. Frydman, Phys. Rev. Lett. 108, 177006 (2012).

[14] Y. Noat, V. Cherkez, C. Brun, T. Cren, C. Carbillet, F. Debontridder, K. Ilin, M. Siegel, A. Semenov, H.-W. Hübers, and D. Roditchev, Phys. Rev. B 88, 014503 (2013).

[15] C. Brun, T. Cren, and D. Roditchev, Supercond. Sci. Technol. 30, 013003 (2017).

[16] A. Ghosal, M. Randeria, and N. Trivedi, Phys. Rev. B 65, 014501 (2001).

[17] Y. Dubi, Y. Meir, and Y. Avishai, Nature (London) 449, 876 (2007).

[18] L. B. Ioffe and M. Mezard, Phys. Rev. Lett. 105, 037001 (2010); M. V. Feigelman, L. B. Ioffe, and M. Mezard, Phys. Rev. B 82, 184534 (2010).

[19] K. Bouadim, Y. L. Loh, M. Randeria, and N. Trivedi, Nat. Phys. 7, 884 (2011).
[20] G. Seibold, L. Benfatto, C. Castellani, and J. Lorenzana, Phys. Rev. Lett. 108, 207004 (2012).

[21] G. Lemarie, A. Kamlapure, D. Bucheli, L. Benfatto, J. Lorenzana, G. Seibold, S. C. Ganguli, P. Raychaudhuri, and C. Castellani, Phys. Rev. B 87, 184509 (2013).

[22] B. Sacépé, T. Dubouchet, C. Chapelier, M. Sanquer, M. Ovadia, D. Shahar, M. Feigel'man, and L. Ioffe, Nat. Phys. 7, 239 (2011).

[23] A. Kamlapure, T. Das, S. Chandra Ganguli, J. B. Parmar, S. Bhattacharyya, and P. Raychaudhuri, Sci. Rep. 3, 2979 (2013).

[24] C. Brun, T. Cren, V. Cherkez, F. Debontridder, S. Pons, D. Fokin, M. C. Tringides, S. Bozhko, L. B. Ioffe, B. L. Altshulerand, and D. Roditchev, Nat. Phys. 10, 444 (2014).

[25] C. Carbillet, S. Caprara, M. Grilli, C. Brun, T. Cren, F. Debontridder, B. Vignolle, W. Tabis, D. Demaille, L. Largeau, K. Ilin, M. Siegel, D. Roditchev, and B. Leridon, Phys. Rev. B 93, 144509 (2016).

[26] P. W. Anderson, J. Phys. Chem. Solids 11, 26 (1959).

[27] M. Ma and P. A. Lee, Phys. Rev. B 32, 5658 (1985).

[28] S. Barabash, D. Stroud, and I.-J. Hwang, Phys. Rev. B 61, R14924 (2000).

[29] S. V. Barabash and D. Stroud, Phys. Rev. B 67, 144506 (2003).

[30] M. Swanson, Y. L. Loh, M. Randeria, and N. Trivedi, Phys. Rev. X 4, 021007 (2014).

[31] T. Cea, D. Bucheli, G. Seibold, L. Benfatto, J. Lorenzana, and C. Castellani, Phys. Rev. B 89, 174506 (2014).

[32] U. S. Pracht, T. Cea, N. Bachar, G. Deutscher, E. Farber, M. Dressel, M. Scheffler, C. Castellani, A. M. Garcia-Garcia, and L. Benfatto, Phys. Rev. B 96, 094514 (2017).

[33] R. W. Crane, N. P. Armitage, A. Johansson, G. Sambandamurthy, D. Shahar, and G. Grüner, Phys. Rev. B 75, 094506 (2007).

[34] E. F. C. Driessen, P. C. J. J. Coumou, R. R. Tromp, P. J. de Visser, and T. M. Klapwijk, Phys. Rev. Lett. 109, 107003 (2012).

[35] P. C. J. J. Coumou, E. F. C. Driessen, J. Bueno, C. Chapelier, and T. M. Klapwijk, Phys. Rev. B 88, 180505 (2013). 
[36] D. Sherman, U. S. Pracht, B. Gorshunov, S. Poran, J. Jesudasan, M. Chand, P. Raychaudhuri, M. Swanson, N. Trivedi, A. Auerbach, M. Scheffler, A. Frydman, and M. Dressel, Nat. Phys. 11, 188 (2015).

[37] B. Cheng, L. Wu, N. J. Laurita, H. Singh, M. Chand, P. Raychaudhuri, and N. P. Armitage, Phys. Rev. B 93, 180511 (2016).

[38] M. Žemlička, P. Neilinger, M. Trgala, M. Rehák, D. Manca, M. Grajcar, P. Szabó, P. Samuely, Š. Gaži, U. Hübner, V. M. Vinokur, and E. Il'ichev, Phys. Rev. B 92, 224506 (2015).

[39] J. Simmendinger, U. S. Pracht, L. Daschke, T. Proslier, J. A. Klug, M. Dressel, and M. Scheffler, Phys. Rev. B 94, 064506 (2016).

[40] N. Bachar, U. Pracht, E. Farber, M. Dressel, G. Deutscher, and M. Scheffler, J. Low Temp. Phys. 179, 83 (2014).

[41] U. S. Pracht, N. Bachar, L. Benfatto, G. Deutscher, E. Farber, M. Dressel, and M. Scheffler, Phys. Rev. B 93, 100503 (2016).

[42] I. Maccari, L. Benfatto, and C. Castellani, Phys. Rev. B 96, 060508(R) (2017).

[43] I. Maccari, L. Benfatto, and C. Castellani, Condens. Matter 3, 8 (2018).

[44] W. Liu, M. Kim, G. Sambandamurthy, and N. P. Armitage, Phys. Rev. B 84, 024511 (2011).
[45] A. Kamlapure, M. Mondal, M. Chand, A. Mishra, J. Jesudasan, V. Bagwe, L. Benfatto, V. Tripathi, and P. Raychaudhuri, Appl. Phys. Lett. 96, 072509 (2010).

[46] M. Mondal, S. Kumar, M. Chand, A. Kamlapure, G. Saraswat, G. Seibold, L. Benfatto, and P. Raychaudhuri, Phys. Rev. Lett. 107, 217003 (2011).

[47] S. Misra, L. Urban, M. Kim, G. Sambandamurthy, and A. Yazdani, Phys. Rev. Lett. 110, 037002 (2013).

[48] J. Yong, T. R. Lemberger, L. Benfatto, K. Ilin, and M. Siegel, Phys. Rev. B 87, 184505 (2013).

[49] I. Hetel, T. R. Lemberger, and M. Randeria, Nat. Phys. 3, 700 (2007).

[50] J. Yong, M. J. Hinton, A. McCray, M. Randeria, M. Naamneh, A. Kanigel, and T. R. Lemberger, Phys. Rev. B 85, 180507 (2012), and references therein.

[51] P. G. Baity, X. Shi, Z. Shi, L. Benfatto, and D. Popović, Phys. Rev. B 93, 024519 (2016).

[52] S. Kirkpatrick, Rev. Mod. Phys. 45, 574 (1973).

[53] G. D. Mahan, Many-Particle Physics (Springer, Berlin, 2000).

[54] S. Banerjee, T. V. Ramakrishnan, and C. Dasgupta, Phys. Rev. B 83, 024510 (2011).

[55] G. Seibold, L. Benfatto, and C. Castellani, Phys. Rev. B 96, 144507 (2017). 